Supporting Information for

\title{
Piperazine derivatives enhance epithelial cell monolayer permeability by increased cell force generation and loss of cadherin structures
}

Shiyuan Zheng $\ddagger$, Kirill Lavrenyuk $\$$, Nicholas G. Lamson, Katherine C. Fein, Kathryn A. Whitehead, Kris Noel Dahl*

2 Supplemental Figures

2 Pages including Cover 


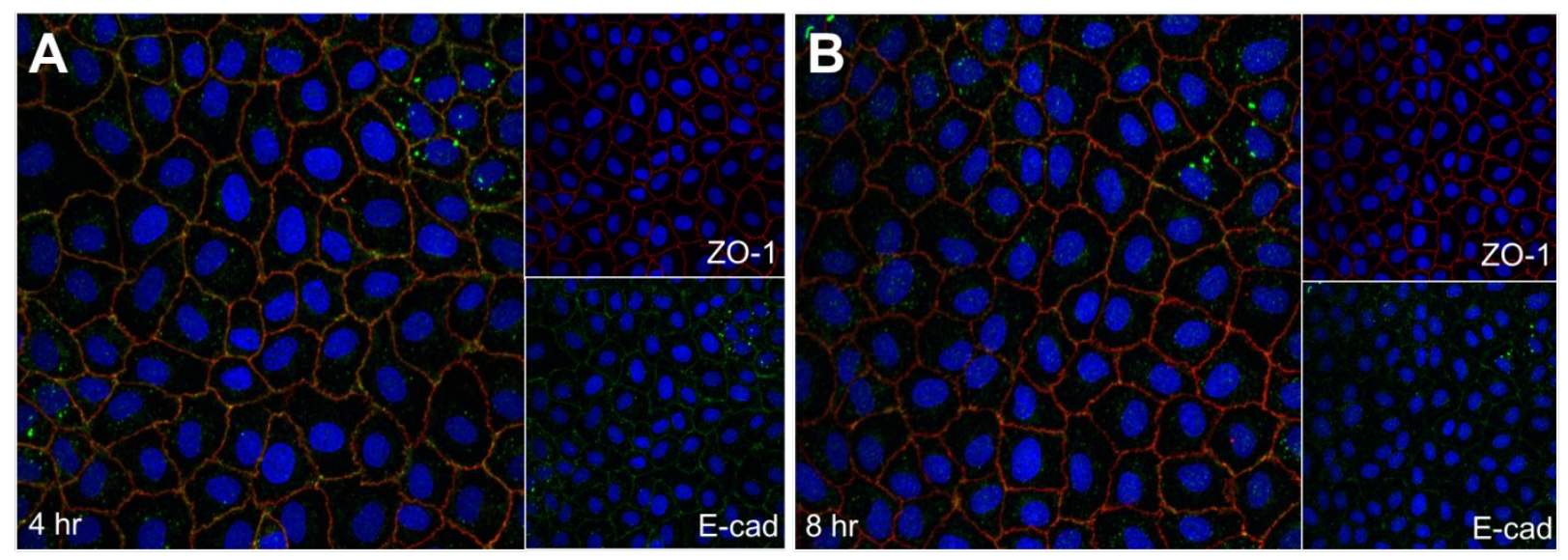

Figures S1. Co-localization of ZO-1 and E-Cadherin after long times with low concentration of 1M4ppz. (A) Co-localization of ZO-1 (red) and E-Cadherin (green) after 4-hours of treatment with $0.1 \mathrm{mM} 1 \mathrm{M} 4 \mathrm{ppz}$ show most structures intact. (B) Co-localization of ZO-1 and E-Cadherin after 8 -hours of treatment with $0.1 \mathrm{mM} 1 \mathrm{M} 4 \mathrm{ppz}$ shows a dramatic loss of E-Cadherin from the plasma membrane. In both cases, ZO-1 is present in the plasma membrane. Co-localization of ECadherin and ZO-1 decreases significantly after 8-hours of treatment relative to the 4-hour time point and the vehicle control.
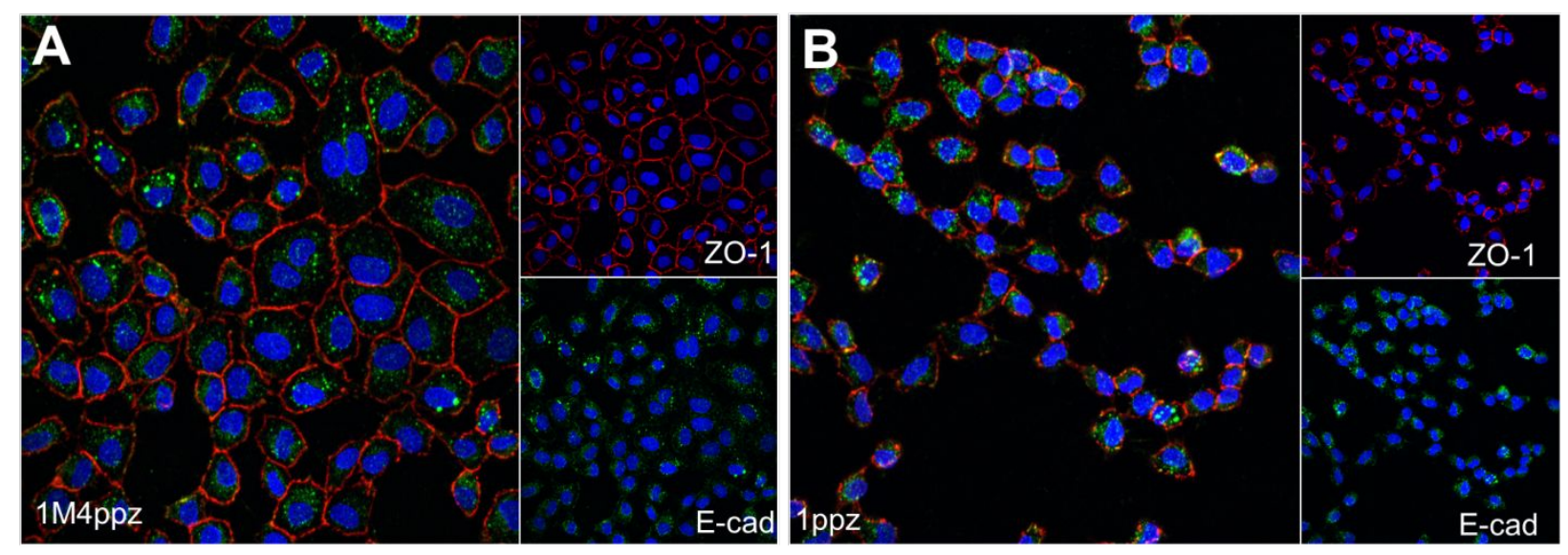

Figure S2. Co-localization of ZO-1 and E-Cadherin after short time with high concentration with piperazine derivatives. (A) Co-localization of ZO-1 (red) and E-Cadherin (green) post 1-hour treatment with 10mM 1M4ppz. (D) Co-localization of ZO-1 and E-Cadherin post 1-hour treatment with $1.0 \mathrm{mM}$ ppz. In both cases, ZO-1 staining is visible at higher concentrations on the cell membrane than E-Cadherin, suggesting a temporal disassembly of the junction where ECadherin is displaced before $\mathrm{ZO}-1$. 